\title{
Rehabilitación mínimamente invasiva con carillas de porcelana: reporte de caso
}

\section{Minimally invasive rehabilitation with porcelain veneers: case report}

Danela Cisneros Boada ${ }^{1 a}$, Victor Montaño Tatés ${ }^{1 a}$, Ana Armas Vega ${ }^{1 b d}$, Santiago Bravomalo Castillo $1^{\text {cd }}$.

\section{RESUMEN}

El presente documento expone la rehabilitación estética dental en el sector anterior maxilar, recuperando las características de los dientes naturales color y forma, a través de, carillas y coronas cerámicas, consiguiendo mejorar la sonrisa en el rostro de la paciente. La intervención mediante procedimientos mínimamente invasivos como las carillas, recupera de forma rápida y con un mínimo margen de error la estética del paciente, sin embargo, un diagnóstico adecuado, planificación de los procesos a seguir, escuchar las necesidades del paciente y priorizarlas, constituye la clave del éxito alcanzado.

Palabras clave: Atención odontológica integral; coronas con frente estético; estética dental; porcelana dental; tratamiento conservador. (Fuente: DeCS BIREME)

\section{ABSTRACT}

The present document exposes the aesthetic dental rehabilitation in the anterior dental sector, recovering the characteristics of natural teeth as color and shape, through ceramic veneers and crowns, getting improve the smile in the face of patient. The intervention by minimally invasive procedures as veneers, recover quickly and with minimum error margin the patient's aesthetics, however, an adequate diagnosis, planning processes, listen the patient's needs and prioritize them, constitute the key to success achieved.

Keywords: Comprehensive dental care; dental esthetics; dental porcelain; dental veneres; conservative treatment. $\underline{\text { Source: }}$ $\underline{\text { MeSH NLM) }}$

${ }^{1}$ Universidad Tecnológica Equinoccial del Ecuador.

a Odontólogo.

b PhD, Operatoria dental.

${ }^{c}$ Rehabilitador Oral.

d Docente

\section{Correspondencia:}

Victor Montaño Tatés

victor18alfonso@hotmail.com
Este es un artículo Open Access distribuido bajo la licencia Creative Commons Atribución-NoComercialCompartir Igual 4.0

\section{વ)}

Citar como: Cisneros D, Montaño V, Armas A. Bravomalo S. Rehabilitación mínimamente invasiva con carillas de porcelana: reporte de caso. KIRU. 2019 ene-mar; 16(1): 32-36. https://doi.org/10.24265/kiru.2019.v16n1.05 


\section{INTRODUCCIÓN}

La estética en odontología permite establecer principios científicos y artísticos que se consideran colectivamente, para crear una sonrisa hermosa con dientes de proporciones intrínsecas agradables entre sí y en armonía con los tejidos gingivales, labios y estructuras faciales ${ }^{(1)}$, como factores determinantes del éxito restaurador de un paciente con desarmonías estéticas dentales.

Las proporciones simetrías dentales están establecidas por medio de proporciones, donde los incisivos centrales son elementos dominantes en la composición de la sonrisa, brindando unidad, fuerza, jovialidad y sensualidad (2), en ese contexto son cruciales la armonía entre altura del labio superior, línea de sonrisa, puntos de contacto, troneras interdentales incisales, troneras interdentales gingivales, posición y apariencia en la región cervical y la posición del cenit gingival ${ }^{(3)}$.

Los nuevos avances en materiales dentales permiten fortalecer las cerámicas, que se caracterizan por ser materiales de restauración indirecta policristalinos que poseen cantidades considerables de feldespato, es decir, cerámicas feldespáticas; compuestas por sílice, litio, aluminio, sodio y potasio como el disilicato de litio, cuyos elementos protésicos se obtienen mediante termo prensado, tecnología CAD/CAM, estratificación o cut-back ${ }^{(4)}$. Obteniendo una gama de cerámicas dentales reforzadas estructuralmente o cerámicas vitrificadas que a su vez es nuestra elección por su porcentaje de supervivencia a 10 años y éxito en estética logrado en el sector anterior, imitando la naturalidad dental, debido a la similitud en sus propiedades ópticas ${ }^{(5)}$.

Con estos antecedentes, el presente artículo expone la rehabilitación estética dental en el sector anterosuperior, reproduciendo las características de los dientes naturales color y forma, a través de carillas, coronas e incrustaciones consiguiendo mejorar la estética, función y autoestima de la paciente.

\section{DESCRIPCIÓN DE LA TÉCNICA}

Paciente femenino de 36 años de edad con antecedentes de cáncer en Tiroides, acude a la Clínica universitaria de la Universidad Tecnológica Equinoccial, Quito, Ecuador; desconforme con el tamaño y forma de sus dientes anteriores maxilares. El examen clínico y radiográfico permite determinar presencia de caries secundaria en el diente 1.1, restauraciones sobre contorneadas, periodontitis crónica moderada localizada y edentulismo parcial Clase I de Kennedy mandibular (Figura 1).

Tras estudio de modelos iniciales obtenidos previamente y análisis de encerado diagnóstico,

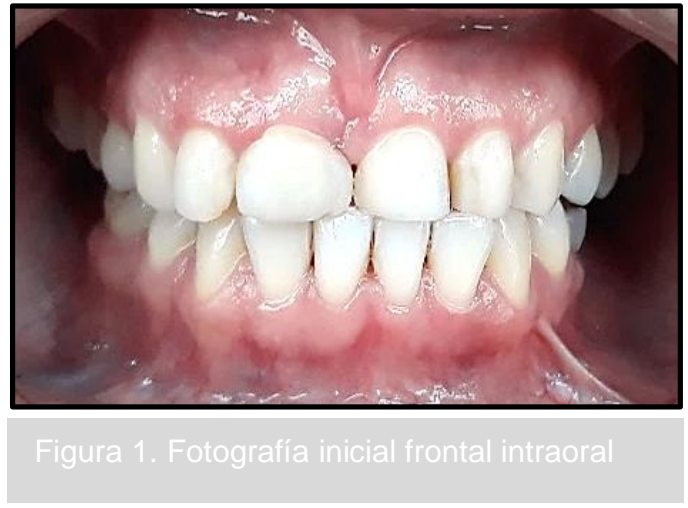

ejecutado en articulador, se discute los planes de tratamiento con la paciente, la cual acepta mediante firma de consentimiento informado para tratamiento clínico y registro fotográfico. Se inicia tratamiento periodontal no quirúrgico, eliminación de restauraciones mal adaptadas y caries secundarias mediante procedimientos directos materiales adhesivos.

El análisis clínico periodontal permitió verificar la necesidad de realizar gingivectomía a nivel de los dientes anteriores maxilares, para mejorar la armonía dental, gingival y facial. Tras 21 días de espera para la cicatrización, los dientes anteriores maxilares recibieron preparación para carilla indirecta, a una profundidad de $0.8 \mathrm{~mm}$, una reducción incisal de 1,5 $\mathrm{mm}$ y $45^{\circ}$ hacia palatino, con fresas diamantadas calibradas y de extremo redondo $(\mathrm{Kg}$-Sorensen, Brasil) en alta velocidad con refrigeración por agua. $\mathrm{E}$ diente 11 debido a las implicaciones en perdida de tejido, fue preparado para corona con un desgaste de $1.5 \mathrm{~mm}$ en todo su contorno; todos los ángulos se redondearon con un disco abrasivo de acabado (Diamond-pro-FGM, Brasil), mediante movimientos intermitentes y definiendo la línea de terminación tipo chaflán continua. La preparación fue realizada mediante matriz de silicona de adición confeccionada a partir del encerado diagnóstico; la impresión de las preparaciones fue realizada mediante técnica de doble hilo para desviar el surco gingival y silicona de adición, enviando al laboratorio junto con la selección del color de acuerdo al sustrato dental para su confección en cerámica vitrificada (IPS e.max Press, Ivoclar Vivadent). Fueron colocados provisionales con resina bis-acrílica elaborados a partir del encerado diagnóstico y matriz de silicona (mock up), hasta concluir la etapa laboratorial.

El análisis de modelos ejecutado previamente determinó la necesidad de nivelar el plano oclusal y confeccionar una prótesis removible inferior,; en ese proceso los dientes 1.6, 1.5 y 4.6 fueron preparados para recibir restauraciones indirectas tipo incrustación overlay de cerómero,. El registro de la preparación fue realizado siguiendo los 
procedimientos establecidos en la técnica, sirviendo como antagonista para el proceso laboratorial.

Tras confección de las restauraciones estéticas anteriores, el mock up fue removido y las superficies dentales desinfectadas; para la valoración en húmedo de cada una de las piezas protésicas, verificándose la adaptación, fonación, parámetros estéticos y satisfacción del paciente. Previo a la cementación, las estructuras protésicas fueron acondicionadas individualmente, con ácido fluorhídrico al $5 \%$ (Condac porcelana, Fgm) durante $20 \mathrm{~s}$ seguido de lavado por 1 minuto, aplicación activa de ácido fosfórico al 37\% (3M-ESPE) por $60 \mathrm{~s}$ como agente neutralizante, lavado y secado, colocación de silano (Monobond Plus, Ivoclar-Vivadent) con microaplicador por $60 \mathrm{~s}$. Al mismo tiempo, las superficies de los dientes preparados fueron acondicionadas con ácido fosfórico al $37 \%$ (3M-ESPE), mediante técnica de grabado total, recibiendo dos capas de adhesivo (Heliobond, Ivoclar-Vivadent) evaporando el solvente con una leve aplicación de aire después de la primera capa y cemento resinoso (Variolink Esthetic, Ivoclar Vivadent) posicionando en cada estructura protésica; se fotopolimerizó por $2 \mathrm{~s}$ eliminando los excesos con un pincel, complementado el proceso durante $30 \mathrm{~s}$ por superficie y aplicación de glicerina con una fotopolimerización final para evitar la capa inhibida de oxígeno. Se eliminó residuos del material adhesivo a nivel gingival mediante bisturí e hilo dental; el proceso de cementación de incrustaciones y corona fue ejecutado mediante cemento (Variolink Esthetic, Ivoclar Vivadent) respetando las indicaciones del fabricante y la técnica establecida para el efecto.El control oclusal fue realizado mediante movimientos de desoclusión y lateralidad eliminando toda interferencia; se realizaron recomendaciones de mantenimiento, citas de control y cuidados, además de una placa estabilizadora oclusal, fueron enviadas a la paciente (Figura 2).
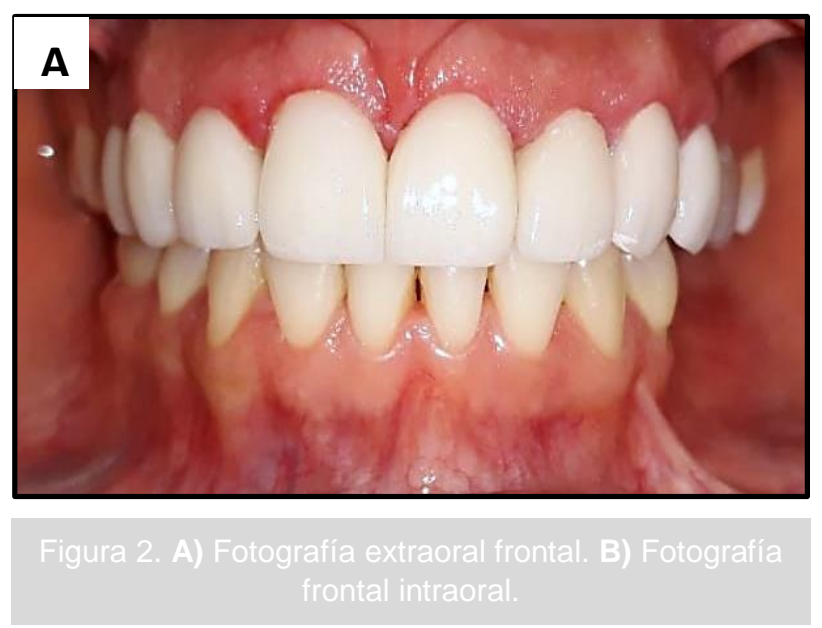

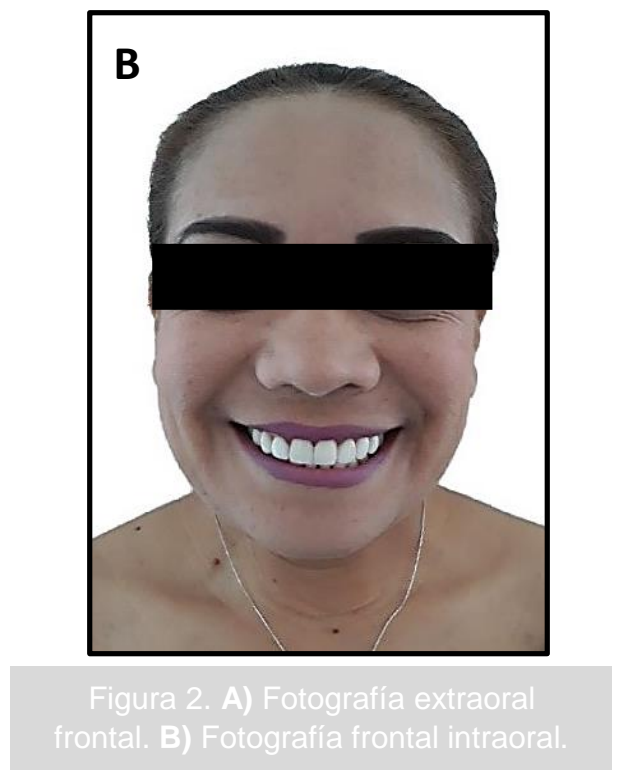

\section{DISCUSIÓN}

La parte estética de la odontología siempre guardará una subjetividad que invite al debate entre clínicos odontólogos, sin embargo, es de vital importancia poder escuchar las necesidad que llevan al paciente a la consulta, en el caso expuesto la confección con materiales indirectos se presentó como la mejor alternativa y permitió una apariencia estética satisfactoria en comparación con materiales directos (6), éxito asociado a sus propiedades físicas y biológicas (7) (3) pese a la existencia de ciertas controversias en cuanto a su longevidad (8).

Las características de las cerámicas dentales, como las ópticas en cuanto a la estabilidad del color, resistencia a la corrosión y resistencia a la fractura hacen de este material una buena opción para restauraciones indirectas, especialmente cuando se requiere funcionalidad y estética ${ }^{(9)}$. Si bien existen variedad de materiales para la elaboración de restauraciones estéticas, los requerimientos del paciente constituyen una guía fundamental en el proceso de selección del material; en el presente caso la paciente en mención requería alta estética, esto permitió optar por emplear restauraciones indirectas elaboradas con porcelana cerámica vitrificada (IPS e.max Press-Ivoclar Vivadent) asociados a su un alto grado de translucidez y estética. Permitiendo restaurar de manera efectiva los dientes anteriores, recuperando funcionalidad como, por ejemplo, una adecuada guía anterior y lateral, importante en la oclusión ${ }^{(10)}$.

El uso clínico del sistema (IPS e.max) asegura la estabilidad clínica de las restauraciones ejecutadas (11) ofreciendo seguridad al clínico y al paciente ${ }^{(12)}$. Sin embargo, exige ciertos cuidados relacionados a su preparación y manejo (13) que requieren ser considerados siempre ${ }^{(14)(9)}$ en relación directa con su 
proceso de adhesión (15), constituyéndose de vital importancia el respeto estricto a los mismos (16) y el empleo de materiales de cementación adecuados ${ }^{(17)}$ (15) siguiendo los protocolos establecidos y las instrucciones del fabricante ${ }^{(18)}$, lo cual lleva a un tratamiento exitoso a largo plazo ${ }^{(19)}$.

Las resinas compuestas con sus evidentes mejoras son una alternativa válida al momento de ejecutar una restauración, sin embargo, la dependencia de la habilidad del operador y las características del propio material (20) (1) constituyen una limitante cuando conseguir alta estética se trata. Si bien, el proceso rehabilitador ejecutado en el sector anterior fue exitoso, el compromiso de la paciente en ejecutar la rehabilitación del sector posterior definitivamente marcará la longevidad de lo realizado. Un acta de compromiso junto con un consentimiento informado claro, detallado, siempre será la herramienta que permita al clínico asegurarse de que lo realizado pueda ser mantenido.

El diagnóstico y la planificación del tratamiento odontológico protésico adecuados, la habilidad y conocimiento del clínico, junto con el arte de escuchar las necesidades del paciente, constituyen el éxito predecible de la rehabilitación mínimamente invasiva del sector anterior, brindando al paciente funcionalidad y armonización dentofacial, mediante un tratamiento integral favorable a largo plazo, siguiendo las indicaciones de mantenimiento partidas por el profesional.

Contribuciones de autoría: DCB, VMT, AAV, SBC diseñaron el reporte de caso, recopilaron, analizaron los datos. Redactaron y aprobaron el artículo todos los autores.

\section{Fuente de financiamiento:}

Autofinanciado.

\section{Conflicto de intereses:}

No existe conflicto de intereses en este manuscrito. Si existiera, sería declarado en este documento y /o explicado en la página del título.

\section{REFERENCIAS}

1. Kosyfaki P, del Pilar MM, Strub JR. Relationship between crowns and the periodontium: a literature update. Quintessence Int. 2010; 41(2):109-126.

2. Viswambaran M. Londhe SM, Kumar, V. Conservative and esthetic management of diastema closure using porcelain laminate veneres. Med J Armed Forces India. 2015; $71(2): 581-585$.
3. Liu X, Zhang Y, Zhou Z, Ma S. Retrospective study of combined splinting restorations in the aesthetic zone of periodontal patients. 2016; 220(5):241-247.

4. Pereira DD. Analysis of marginal adaptation of porcelain laminate veneres produced by computer-aided design/computer-assisted manufacturing technology: Apreliminary in vitro study. Int J Prosthodont. 2018; 31(4): 346-348.

5. EISayad II. Color and translucency of finished and unfinished esthetic restorative materials after staining and bleaching. Saudi Dent J. 2018; 30(3):219-225.

6. Korkut B, Yanikoglu F, Gunday M. Direct composite laminate veneers: three case reports. $\mathrm{J}$ Dent Res Dent Clin Dent Prospects. 2013; 7:105-111.

7. Demarco FF, Collares K, Coelho FH, Correa MB, Cenci MS, Moraes RR, et al. Anterior composite restorations: A systematic review on long-term survival and reasons for failure. Dent Mater. 2015; 31:1214-1224.

8. Re D, Augusti G, Amato M, Riva G, Augusti, D. Esthetic Rehabilitation of Anterior Teeth with Laminates Composite Veneers. Case Rep Dent. 2014. 2014:849273.

9. da Cunha LF, Pedroche LO, Gonzaga CC Furuse AY. Esthetic, occlusal, and periodontal rehabilitation of anterior teeth with minimum thickness porcelain laminate veneers. J Prosthet Dent. 2014; 112(6):1315-8.

10. Zhou TF, Wang XZ, Liu JY, Sun Q, Wang XK. Preliminary clinical evaluation of the esthetic effect of deep discolored anterior teeth restored with zirconia veneres. 2016; 48(6):1055-1059.

11. Dumfahrt $H$, Schaffer $H$. Porcelain laminate veneers. A retrospective evaluation after 1 to 10 years of service: Part II--Clinical results. Int $\mathrm{J}$ Prosthodont. 200; 13(1):9-18.

12. Frese $C$, Staehle HJ, Wolff D. The assessment of dentofacial esthetics in restorative dentistry: a review of the literature. J Am Dent Assoc. 2012; 143:461-466.

13. Petridis HP, Zekeridou A, Malliari M, Tortopidis D, Koidis P. Survival of ceramic veneers made of different materials after a minimum follow-up period of five years: a systematic review and meta-analysis. Eur J Esthet Dent. 2012; 7(2):138-152.

14. Layton DM, Walton TR. The up to 21-year clinical outcome and survival of feldspathic porcelain veneers: accounting for clustering. Int $\mathrm{J}$ Prosthodont. 2007; 25: 604-612.

15. Peumans $M$, Van Meerbeek B, Lambrechts $P$, Vanherle G. Porcelain veneers: a review of the literature. Rev ADM. 2000; 28(3):163-177.

16. Cho SH, Lopez A, Berzins DW, Prasad S, Ahn KW. Effect of Different Thicknesses of Pressable Ceramic Veneers on Polymerization of Light- 
cured and Dual-cured Resin Cements. J Contemp Dent Pract. 2015; 16(5): 347-352.

17. Farias $A$, Gomes $C F$, Sánchez $A$, Sánchez $A$, Vilanova LR. Esthetic Rehabilitation of the Smile with No-Prep Porcelain Laminates and Partial Veneers.Case Rep Dent. 2015.

18. Federizzi L, Gomes ÉA, Báratro SS, BarattoFilho F, Bacchi A, Spazzin AO. Use of Feldspathic Porcelain Veneers to Improve Smile Harmony: A 3-Year Follow-up Report. Braz Dent. 2016; 27(6): 767-774.
19. Burke FT. Survival rates for porcelain laminate veneers with special reference to the effect of preparation in dentin: a literature review. RODE. 2015; 24(4): 257 - 265.

20. Gresnigt MM, Özcan M, Carvalho M, Lazari P, Cune MS, Razavi P, et al. Effect of luting agent on the load to failure and accelerated-fatigue resistance of lithium disilicate laminate veneers. Dent Mater. 2017 Dec; 33(12):1392-1401. 